\title{
No Evidence for the Induction of Brown Algal Chemical Defense by the Phytohormones Jasmonic Acid and Methyl Jasmonate
}

\author{
Theresa Wiesemeier • Karsten Jahn • Georg Pohnert
}

Received: 30 April 2008 /Revised: 21 October 2008 / Accepted: 27 October 2008 /Published online: 20 November 2008

(C) Springer Science + Business Media, LLC 2008

\begin{abstract}
Induced chemical defense reactions are widespread in marine brown algae. Despite the evidence that the biosynthesis of defense metabolites can be up-regulated upon herbivory, we do not know how this regulation of biosynthetic pathways to secondary metabolites is achieved in brown algae. In higher plants, the phytohormone jasmonic acid (JA) is crucial for the mediation of induced chemical defenses, and several findings of this metabolite from marine sources have been reported. We tested the hypothesis that JA or related metabolites play a role in induced brown algal defense. Quantification of oxylipins with a detection limit around $20 \mathrm{ng} \mathrm{g}^{-1}$ algal tissue did not reveal the presence of JA in the seven examined brown algal species Dictyota dichotoma, Colpomenia peregrina, Ectocarpus fasciculatus, Fucus vesiculosus, Himanthalia elongata, Saccharina latissima (formerly Laminaria saccharina), and Sargassum muticum. Moreover, treatment with ecologically relevant concentrations of JA and methyl jasmonate did not lead to a significant change in the profile of medium- and non-polar metabolites of the tested algae. Only when high concentrations of $\geq 500 \mu \mathrm{g} \mathrm{ml}^{-1}$ medium of the phytohormones were applied that a metabolic response which could be attributed to unspecific stress was observed.
\end{abstract}

\section{T. Wiesemeier $\cdot$ K. Jahn $\cdot$ G. Pohnert}

Laboratory of Chemical Ecology/Institutes of Chemical

Sciences and Engineering,

École Polytechnique Fédérale de Lausanne (EPFL),

1015 Lausanne, Switzerland

\section{Present address:}

T. Wiesemeier $\cdot$ G. Pohnert $(\bowtie)$

Bioorganic Analytics, Institut für Anorganische und Analytische

Chemie, Friedrich-Schiller-Universität Jena,

Lessingstr. 8,

07743 Jena, Germany

e-mail: georg.pohnert@uni-jena.de
Bioassays with $D$. dichotoma that focused on medium- and non-polar compounds confirmed the lack of a biological role of JA and methyl jasmonate in the induction of algal induced chemical defenses. The phytohormone-treated samples did not exhibit any increased defense potential towards the amphipod Ampithoe longimana and the isopod Paracerceis caudata. JA and related phytohormones, known to be active in higher plants, thus appear to play no role in brown algae for induction of the defense chemicals studied here.

Keywords Dictyota dichotoma . Dictyotales . Induced defense $\cdot$ Metabolic profiling $\cdot$ Metabolomics . Terpenes · Jasmonic acid · Methyl jasmonate · Brown algae . Phytohormones

\section{Introduction}

Despite large differences between habitats of seaweeds and land plants, both types of plants suffer similar constraints, such as competition for living space, nutrients, and light. Costs caused by infections and herbivores also are comparable (Pavia et al. 2002). Both higher plants and seaweeds have developed efficient chemical defense systems that overcome these limitations (Cronin and Hay 1996; Kupper et al. 2002; Pohnert 2004). In general, plant defense reactions can be categorized into constitutive, activated, and inducible mechanisms (Pohnert 2004). While the first two strategies are based on metabolites, which are either permanently stored in the tissue or rapidly transformed into more reactive species upon wounding, induced defenses rely on de novo production of defense metabolites that target herbivores or pathogens. As a result, induced defenses allow higher plants and algae to avoid the costly 
permanent production and storage of defense metabolites, and resources required for their biosynthesis are invested only in times of increased herbivore or pathogen pressure (Karban and Baldwin 1997).

Despite several observations of induced defense in brown algae (Cronin and Hay 1996; Pavia and Toth 2000; Hemmi et al. 2004; Macaya et al. 2005; Molis et al. 2006), there is an ongoing debate about how the induction of the de novo biosynthesis of defensive metabolites is regulated (Tarakhovskaya et al. 2007). In higher plants, hormones such as jasmonic acid (JA) and methyl jasmonate (MeJA), as well as external elicitors and continuous mechanical tissue disruption, can orchestrate the induction of chemical defenses after mechanical wounding, herbivore damage, or pathogen attack (Karban and Baldwin 1997; Baldwin et al. 2002; Wittstock and Gershenzon 2002; Mithöfer et al. 2005; Garcia-Brugger et al. 2006; Wasternack 2007). JA and MeJA have been identified in red algae (Rhodophyta) as well (Krupina and Dathe 1991; Bouarab et al. 2004; Tarakhovskaya et al. 2007). JA influences growth and development in the green microalga Chlorella vulgaris (Czerpak et al. 2006). Moreover, JA and MeJA have a positive effect on the tolerance of the green microalga Scenedesmus incrassulatus to stress temperatures and infections (Christov et al. 2001), but the occurrence of these metabolites in both algae has not been verified. The question whether JA and its derivatives play a role in the induced defense reactions of the red macroalga Chondrus crispus was addressed by Bouarab et al. (2004). They suggested a role of this metabolite as a hormone comparable to that found in higher plants. However, JA and MeJA seem not to be ubiquitous in red algae since Gracilaria chilensis does not contain detectable amounts even when heavily challenged with pathogens (Wiesemeier and Pohnert, personal observation).

While several reports on the potential hormonal role of $\mathrm{JA}$ and MeJA in red and green algae are available, the occurrence and significance in brown algae has been poorly addressed. Arnold et al. (2001) reported that exposure of the common rockweed Fucus vesiculosus to MeJA during periods of tidal emergence causes induction of polyphenolic chemical defenses. Since timing and magnitude of this induced increase in phlorotannin concentration was similar to that caused by herbivory, the question arose whether jasmonates or their oxylipin relatives are natural elements of antiherbivore responses in Fucus.

In this study, we report a series of experiments on the occurrence and potential influence of JA and its derivative MeJA on the formation of medium- and non-polar secondary metabolites in different brown alga species. In addition, the JA mimic coronalone (Schüler et al. 2004) was investigated to exclude artefacts due to metabolization of JA in the medium. Special attention was given to the brown alga Dictyota dichotoma for two reasons: (a) this alga is known to exhibit induced defense (Cronin and Hay, 1996), and (b) it is rich in bioactive terpenoids (Fattorusso et al. 1976; Teixeira et al. 2001; Barbosa et al. 2004; Kim et al. 2006), a compound class often involved in induced defense of higher plants. The chemical profiling was paired with bioassays that used herbivores of algae; these assays were conducted to study the role of JA and MeJA as hormones involved in the production of medium- and nonpolar antiherbivore metabolites of brown algae.

\section{Methods and Materials}

Test Organisms The brown algae D. dichotoma, Colpomenia peregrina, Ectocarpus fasciculatus, F. vesiculosus, Himanthalia elongata, Saccharina latissima (formerly Laminaria saccharina), and Sargassum muticum were collected on the Atlantic coast in Roscoff, France and transferred to the laboratory within 2 days. D. dichotoma was collected from June until August 2004 as well as in March 2006. All other brown algae were collected exclusively on the later date. Algae were kept at $16^{\circ} \mathrm{C}$ under a 14:10 h L/D regime in artificial seawater. Artificial seawater was prepared by dissolving $33 \mathrm{~g}^{-1}$ Instant Ocean (Aquarium Systems, Sarrebourg, France) in distilled water.

The amphipod Ampithoe longimana and the isopod Paracerceis caudata are widely distributed along the Atlantic coast of North America. Test animals for these assays were collected in Morehead City, NC (USA) and airshipped to the laboratory, where they were kept in artificial seawater under identical culture conditions as the brown algae.

Induction Experiments Racemic MeJA was provided by R. Kaiser, Givaudan-Roure, Dübendorf, Switzerland; racemic JA was synthesized by base-mediated saponification of MeJA and subsequently purified by column chromatography. Coronalone (Schüler et al. 2004) was provided by W. Boland, Max-Planck-Institute for Chemical Ecology, Jena, Germany. Linolenic acid (LEA) was obtained from Sigma/ Aldrich, Düren, Germany. For induction experiments, defined pieces of freshly collected algae $(D$. dichotoma, C. peregrina, E. fasciculatus, F. vesiculosus, H. elongata, $S$. latissima, and S. muticum, $80-400 \mathrm{mg}$ ) were kept in $20 \mathrm{ml}$ filtered seawater that contained different amounts of potential elicitors (added from $100 \mathrm{mg} \mathrm{ml}^{-1}$ stock solutions in methanol). Solvent controls were run in parallel. $D$. dichotoma was treated with $0.1,0.5$, and $1 \mathrm{mg} \mathrm{ml}^{-1} \mathrm{JA}$ and LEA, respectively, and with 0.1 and $0.5 \mathrm{mg} \mathrm{ml}^{-1} \mathrm{MeJA}$, where solubility problems occurred at higher concentration. Incubations were performed for 2, 24, and $48 \mathrm{~h}$. The other algae were treated with $0.5 \mathrm{mg} \mathrm{JA}$ and MeJA, respectively, 
for 12 and $48 \mathrm{~h}$. All treatments were performed at least in triplicate. After the induction period, algal pieces were rinsed carefully with seawater, blotted dry with a paper towel, weighed, wrapped in aluminum foil, shock-frozen in liquid nitrogen, and kept at $-20^{\circ} \mathrm{C}$ until further workup.

For bioassays, D. dichotoma was induced with $0.5 \mathrm{mg}$ $\mathrm{ml}^{-1} \mathrm{JA}$, MeJA, or LEA (added from $100 \mathrm{mg} \mathrm{ml}^{-1}$ stock solutions in methanol) in seawater for $30 \mathrm{~h}$. A solvent control was run in parallel. After the incubation period, the algae were rinsed carefully with seawater, blotted dry with a paper towel, and used for the preparation of artificial food as described below.

Profiling of Acidic Oxylipins Bearing Aldehyde or Ketone Structural Elements and Screening for Jasmonic Acid Profiling of oxylipins in D. dichotoma was accomplished by using a solid phase extraction protocol optimized by Schulze et al. (2006). Briefly, alga pieces (1-3 g) were covered with $5 \mathrm{ml} \mathrm{MeOH}$ that contained $0.05 \%$ 2,6-di-tertbutyl-4-methylphenol, followed by the addition of $O$ (2,3,4,5,6-pentafluorobenzyl)hydroxylamine hydrochloride (3 $\mathrm{ml}, 0.05 \mathrm{M}$ in methanol). To monitor extraction efficiency, $\left[9,10-{ }^{2} \mathrm{H}_{2}\right]$-dihydrojasmonic acid (150 ng) was added. The mixture was homogenized for 5 min under an inert argon atmosphere with a high-performance dispenser at 24,000 rpm (Ultra-Turrax T 25, IKA-Werk, Staufen, Germany). Subsequent acidification with diluted $\mathrm{HCl}$ $(\mathrm{pH} 3)$ was followed by extraction of the $\mathrm{MeOH} /$ water phase with hexane $(3 \times 10 \mathrm{ml})$. The remaining polar phase was acidified again by the addition of $4 \mathrm{ml}$ of diluted $\mathrm{HCl}$ $(\mathrm{pH} 3)$ to enhance phase separation and extracted with dichloromethane $(3 \times 10 \mathrm{ml})$. The organic fractions were passed separately through preconditioned Chromabond $\mathrm{NH}_{2}$ cartridges (0.5 g, aminopropyl, Macherey-Nagel, Düren, Germany). After sample loading, the cartridges were washed with 2-propanol/ $\mathrm{CH}_{2} \mathrm{Cl}_{2}(5 \mathrm{ml}, 2: 1, v / v)$ and eluted with diethyl ether/formic acid $(10 \mathrm{ml}, 98: 2, v / v)$. The solvent was removed at RT by a stream of argon. For derivatization of the carboxyl groups, samples were treated with an ethereal solution of diazomethane $(1 \mathrm{ml})$. After 2 min, excess of diazomethane and the solvent was removed by a stream of argon, and the residue was dissolved in dichloromethane $(30 \mu \mathrm{l})$. Samples were analyzed by using a Finnigan TraceMS coupled to a Finnigan GCQ gas chromatograph/mass spectrometer (Thermo-Finnigan, Waltham, MA, USA) equipped with an Alltech EC5 capillary column (15 $\mathrm{m} \times 0.25 \mathrm{~mm}, 0.25 \mu \mathrm{m}$, Alltech, Hamburg, Germany). The ions at $m / z=399$ were used to monitor JA and $m / z=481$ to monitor 12-oxophytodienoic acid (OPDA).

Profiling of Terpenoids and Other (Semi)volatiles For metabolic profiling of terpenoids and other (semi)volatiles, frozen samples of the seven brown algal species were ground in a mortar and extracted directly with dichloromethane $(3 \times 0.5-1 \mathrm{ml})$. The combined organic phases were dried over $\mathrm{Na}_{2} \mathrm{SO}_{4}$, and the solvent was removed under a stream of nitrogen. Samples were taken up with dichloromethane containing $20 \mu \mathrm{g} \mathrm{ml}^{-1}$ laurylaldehyde as standard to give a final volume corresponding to $2 \mathrm{ml}$ solvent per gram alga. These samples were submitted directly in split mode to a Finnigan TraceMS coupled to a Finnigan ITS gas chromatograph equipped with an Alltech EC5 capillary column $(15 \mathrm{~m} \times 0.25 \mathrm{~mm}, 0.25 \mu \mathrm{m}$, Alltech, Hamburg, Germany). The temperature program was $50^{\circ} \mathrm{C}(2 \mathrm{~min})$ then ramped at $10^{\circ} \mathrm{C} \mathrm{min}^{-1}$ to $280^{\circ} \mathrm{C}(2 \mathrm{~min})$. Peaks were assigned by retention time and verified by mass spectra. In cases of poor chromatographic resolution, characteristic ion traces were used for the relative quantification.

Metabolic Profiling of Methylated Extracts Frozen samples were ground and extracted as described above. $\left[{ }^{2} \mathrm{H}_{27}\right]-$ myristic acid $\left(0.1 \mu \mathrm{l}\right.$ of a $10 \mathrm{mg} \mathrm{ml}^{-1}$ stock solution in $\mathrm{MeOH}$ per milligram alga) was added as standard prior to extraction. After removal of the solvents under a stream of nitrogen, an excess of trimethylsilyldiazomethane solution (1 $\mathrm{ml}$ of a $2 \mathrm{M}$ solution in diethylether, Sigma/Aldrich, Buchs, Switzerland) and $10 \mu \mathrm{MeOH}$ was used for derivatization. After $1 \mathrm{~h}$, the excess derivatization reagent was removed under a stream of nitrogen, and $500 \mu \mathrm{l}$ dichloromethane was added. These samples were submitted directly in split mode for gas chromatography (GC)/mass spectroscopy (MS) analysis. Profiling of derivatized samples was performed as described above (temperature program of the $\mathrm{GC}, 60^{\circ} \mathrm{C}(2 \mathrm{~min})$, then ramped at $20^{\circ} \mathrm{C} \mathrm{min}{ }^{-1}$ to $140^{\circ} \mathrm{C}$, then at $5^{\circ} \mathrm{C} \mathrm{min}{ }^{-1}$ to $250^{\circ} \mathrm{C}$ and at $20^{\circ} \mathrm{C} \min ^{-1}$ to $\left.300^{\circ} \mathrm{C}(2 \mathrm{~min})\right)$. Fatty acid methyl esters were identified by comparison of retention time and mass spectra with commercially available standards.

LC/MS Profiling For metabolic profiling of more polar metabolites, frozen samples were ground in a mortar and extracted directly with methanol or a 1:1 mixture of methanol and water ( $3 \mathrm{ml}$ per $400 \mathrm{mg}$ alga). Samples were centrifuged and the supernatant was submitted directly to liquid chromatography (LC)/MS analysis. HPLC/MS profiling was done on a Finnigan LCQ connected to an Agilent 1100 System equipped with a Grom-Sil 120 ODS-3 CP column $(125 \times 2 \mathrm{~mm}$, particle size $3 \mu \mathrm{m}$, Grom, RottenburgHailfingen, Germany). Samples were run twice with APCI and ESI ionization, respectively. A gradient of water $/ 0.5 \%$ acetic acid (solvent $\mathrm{A}$ ) and acetonitrile/0.5\% acetic acid (solvent B) was used at a total flow rate of $0.2 \mathrm{ml} \mathrm{min}^{-1}$ : solvent B 10\% (7 min), 27-37 min 100\% B, and $38.50 \mathrm{~min}$ $10 \% \mathrm{~B}(9.5 \mathrm{~min})$.

Peaks were assigned by retention time in the base peak monitoring mode and verified by mass spectra. In cases of 
poor chromatographic resolution, characteristic ion traces were used for relative quantification.

Evaluation of Profiling Experiments Each treatment was replicated at least in triplicate. For profiling, all peaks $>5 \%$ were quantified and normalized to the internal standard. Differences between induced and uninduced samples were evaluated after calculating the standard deviation using Microsoft Excel. If ambiguous results were observed, additional control/induction pairs were generated and consistency was verified. Minor peaks were evaluated by visual pattern comparison. Quantification was done by using peak areas of characteristic ion traces (LC/MS) or TIC (GC/ MS). Peaks were considered different in control and treatment when $>10 \%$ difference of the respective integrals was observed in all three measurements of the control and of the treated sample. For major peaks, differences were evaluated additionally based on standard deviation.

Bioassays Agar (Aldrich, Seelze, Germany)-based food was prepared by using the methods described in detail by Wiesemeier et al. (2007). Freshly collected D. dichotoma were put into $200 \mathrm{ml}$ culture containers and either treated with $500 \mu \mathrm{g} \mathrm{ml}^{-1}$ of JA, MeJA, or LEA for $30 \mathrm{~h}$, or kept as solvent control. Subsequently, the algae were washed carefully with excess seawater to avoid contamination of the food with inducing reagents. These algae were freezedried, finely powdered, and subsequently incorporated into an agar matrix prepared by mixing $0.72 \mathrm{~g}$ of agar with $36 \mathrm{ml}$ distilled water and dissolving the mixture by heating in a microwave. Subsequently, $2 \mathrm{~g}$ of powdered alga was added, and the mixture was shaken vigorously to homogenize. This mixture was poured into $25 \mathrm{ml}$ plastic centrifuge tubes (Falcon, Franklin Lakes, NY, USA). The tubes were inverted and cooled to room temperature. After agar solidification, the falcon tube was opened and the agar was cut to give pieces of a quarter of a ca. 2-mm-thick agar disc.

We tested responses of $A$. longimana and $P$. caudata to agar-based food with induced and uninduced $D$. dichotoma. Feeding assays were performed in 12-well ice cube containers, each well filled with $20 \mathrm{ml}$ of sea water $\left(18^{\circ} \mathrm{C}\right)$. One amphipod per vessel could choose between one control and one induced food pellet that were arranged on specified sides of the assay container. During $25 \mathrm{~min}$, we noted every 5 min on which type of food each test animal was present. This behavioral association assay is a valid surrogate for feeding assays because these test animals tend to live on, and thus associate spatially with the foods that they consume (Cruz-Rivera and Hay 2003). To statistically evaluate food choice, we used the Wilcoxon test for paired samples.

\section{Results}

Oxylipin Profiling The oxylipin profiling method used allows the detection of JA in concentrations down to $20 \mathrm{ng} \mathrm{g}^{-1}$ fresh material. Nevertheless, JA was not found in the brown alga D. dichotoma (Fig. 1). Moreover, the

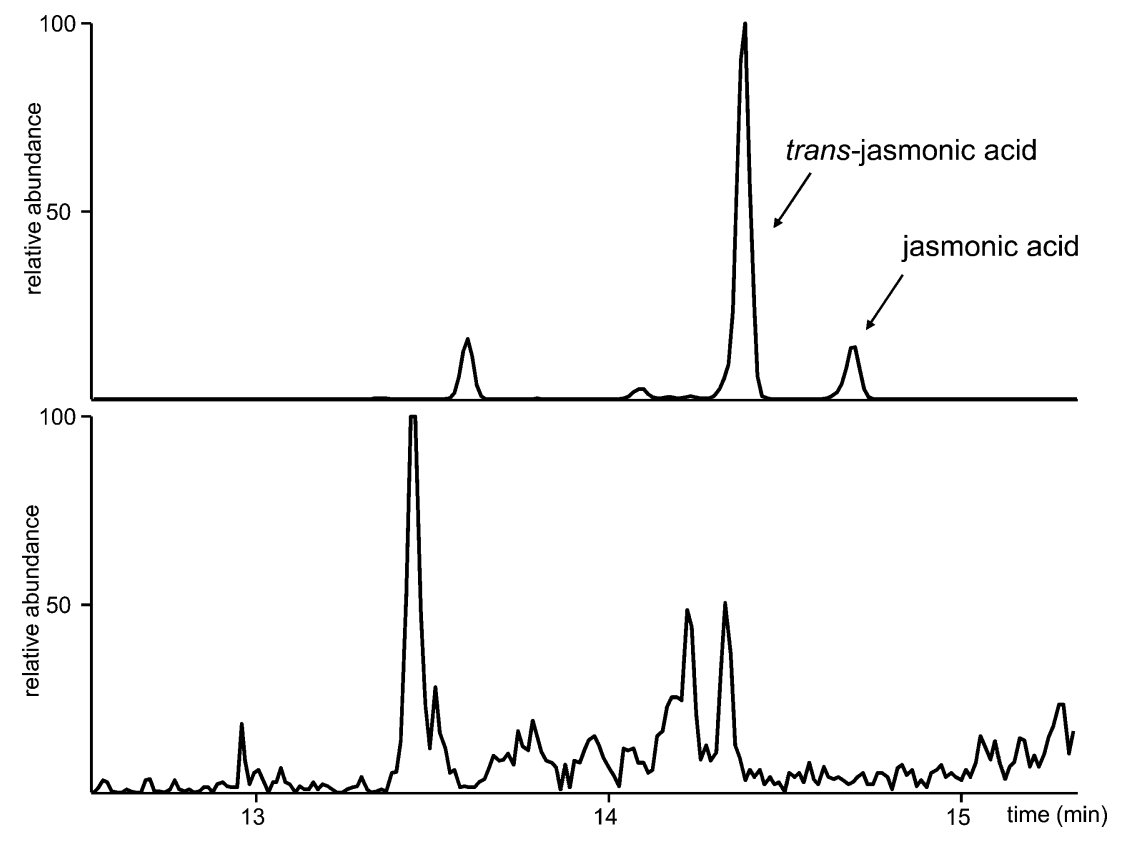

Fig. 1 GC/MS (extracted single ion chromatogram $\mathrm{m} / z=399$ corresponding to M-20 due to a loss of HF) separation of derivatized jasmonic acid (upper trace) and the oxylipin extract of Dictyota dichotoma (lower trace). Spectra were recorded by using chemical ionization with methane as reagent gas in the negative mode 
biosynthetic precursor 12-oxophytodienoic acid also could not be detected (data not shown). In parallel experiments, samples from higher plants (Phaseolus lunatus) were run as positive control. As expected, JA and OPDA were detected in these controls (Schulze et al., 2006).

Metabolic Profiling Induction experiments with JA, MeJA, and LEA (control) were run with freshly collected samples to verify whether significant changes of the metabolic profile of the algae occur in response to the putative hormones. Metabolites with a broad biosynthetic spectrum that included terpenes, phenolics, lipids, and fatty acids were covered by using general methods of metabolic profiling (Weckwerth et al. 2005).

No significant metabolic response was observed when the putative hormones were administered in the seawater in concentrations below $500 \mu \mathrm{g} \mathrm{ml}{ }^{-1}$ to the algae $D$. dichotoma, C. peregrina, E. fasciculatus, F. vesiculosus, $H$. elongata, S. latissima, or S. muticum. In similar experiments with higher plants, significant up-regulation of secondary metabolites can be detected (Boland et al. 1995). In Fig. 2, a GC/MS comparison of trimethylsilyldiazomethane-methylated organic extracts of $D$. dichotoma is shown. Similar chromatograms were used for the evaluation of metabolic differences of terpenoids and other (semi)volatiles. Peak lists were generated from these chromatograms. If inconsistent results were obtained, reevaluation of the peaks was performed by using single ion monitoring, or a second set of replicates was generated.
None of the carefully evaluated peak lists of all seven algal species showed significant differences between treatment and control. LC/MS profiling of JA-treated samples was evaluated by comparison of extracts from JA-treated and untreated algae. Peaks were evaluated in base peak monitoring mode and verified with single ion monitoring. In no evaluated case (peaks with more than $5 \%$ intensity) could a statistical significant up- or down-regulation of signals be observed.

Only if high amounts $\geq 500 \mu \mathrm{g} \mathrm{ml}^{-1}$ of potential elicitors added over a prolonged period of more than $24 \mathrm{~h}$ was a metabolic response observed (Fig. 2). With the exception of S. muticum, all investigated algae released fatty acids, but their nature and amount varied strongly between experiments. In the experiments with $D$. dichotoma, up to 500fold up-regulation of free palmitic acid, palmitoleic acid, and oleic acid was observed. Besides these metabolites, strongly varying amounts of saturated fatty acids, such as lauric (C12:0), myristic (C14:0), stearic (C18:0), arachidic (C20:0), and behenic acid (C22:0), as well as unsaturated fatty acids, such as stearidonic (C18:4), $\alpha$-linolenic (C18:3), arachidonic (C20:4), and eicosapentaenoic acid (C20:5) were released by all investigated algae with the exception of $S$. muticum. This metabolic response was unspecific and could be triggered with high concentrations of JA, MeJA, and LEA. During these treatments, algae were heavily stressed and depigmentation was observed after more than $48 \mathrm{~h}$. The solvent control did not lead to comparable metabolic and stress reactions.

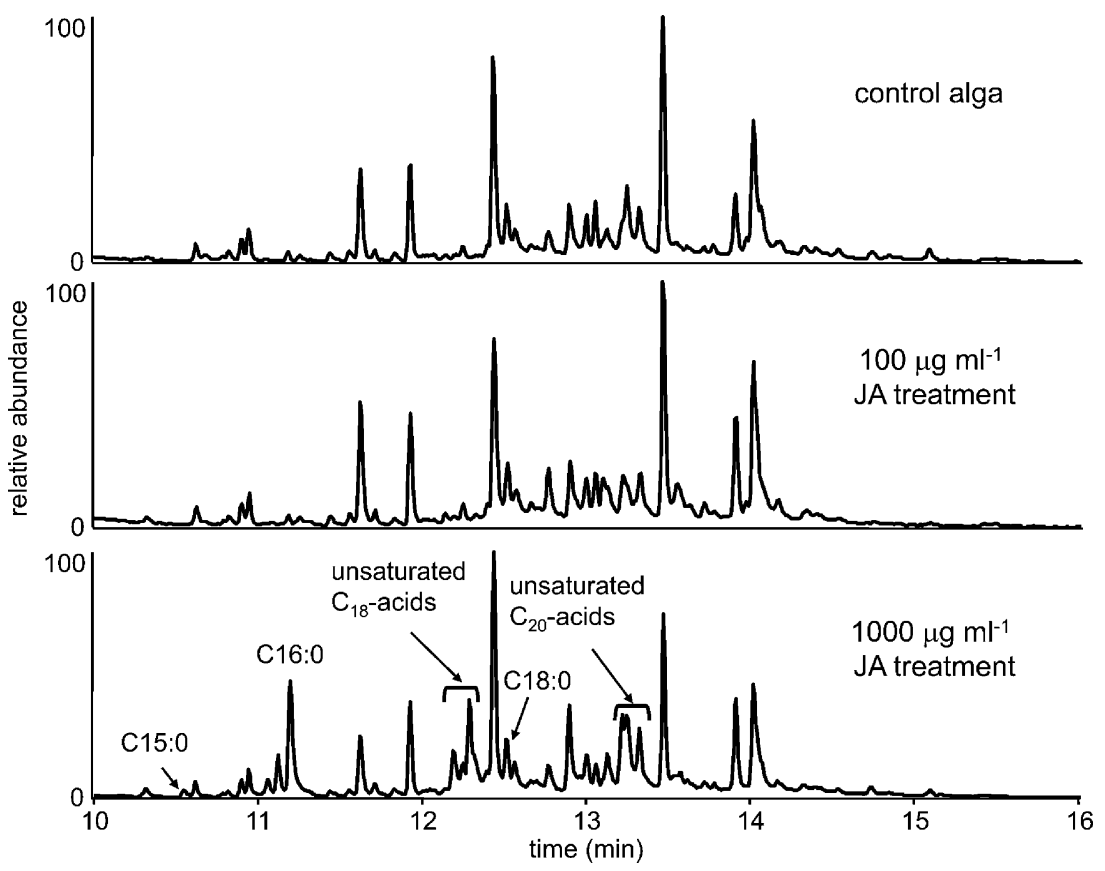

Fig. 2 GC/MS profiling (total ion current) of methylated Dictyota dichotoma extracts. Upper trace: untreated control; middle trace: after incubation with $100 \mu \mathrm{g} \mathrm{ml}^{-1} \mathrm{JA}$; lower trace: after incubation with
$1,000 \mu \mathrm{g} \mathrm{mL} \mathrm{m}^{-1} \mathrm{JA}$. The respective up-regulated fatty acids are indicated. C16:0 palmitic acid, C18:0 stearic acid 
Additional profiling of terpenoids and other semivolatiles of $D$. dichotoma after challenging the algae with coronalone $\left(10,100\right.$, and $\left.1,000 \mu \mathrm{g} \mathrm{ml}^{-1}\right)$ for $24 \mathrm{~h}$ also did not reveal any specific induction of secondary metabolites (data not shown).

Bioassays on the Induction of Chemical Defense Bioassays were performed with two model herbivores, the amphipod A. longimana and the isopod P. caudata (Wiesemeier et al. 2007). Food from control algae was compared with food from algae treated with $500 \mathrm{\mu g} \mathrm{ml}^{-1}$ of the inducing reagents, which corresponds to the lowest concentrations at which a metabolic response was observed. P. caudata associated unselectively with both types of food offered; no preference was detectable for control food nor for JA-, MeJA-, or LEAtreated $D$. dichotoma. In contrast, $A$. longimana selected preferentially $(P=0.01)$ food from algae pre-treated with $500 \mu \mathrm{g} \mathrm{ml}^{-1}$ JA, MeJA, or LEA for $30 \mathrm{~h}$ (Fig. 3).

\section{Discussion}

The brown alga $D$. dichotoma was submitted to a robust metabolic profiling method of oxylipins based on derivatization and detection with gas chromatography/mass spectrometry (Wichard et al. 2005; Schulze et al. 2006). Despite a detection limit well below $20 \mathrm{ng} \mathrm{g}^{-1}$ plant material (Schulze et al. 2006), no traces of JA were found in this algal species (Fig. 1). Given the resting level of JA in plant tissue (around $50 \mathrm{ng} \mathrm{g}^{-1}$ ) and the amounts above $1,300 \mathrm{ng} \mathrm{g}^{-1}$ of JA that can be reached during active induction (Schulze et al. 2006; Adra et al. 2006), it can be excluded that JA concentrations in the investigated brown algae were high enough to induce defense responses triggered by mechanisms that are comparable to those in higher plants. Moreover, 12-oxophytodienoic acid, a biosynthetic precursor of JA that is present in considerable amounts in higher plants, could not be detected in algal extracts (Feussner and Wasternack 2002). Samples from higher plants were run in parallel as positive controls. In these samples, JA and OPDA were detected, thereby providing proof of suitability of the method employed (data on higher plants shown in Schulze et al. 2006). This finding suggests that brown algae lack a higher plant-like pathway towards JA. Nevertheless, it cannot be excluded that some algal species may contain JA or related metabolites.

Because we studied only freshly collected material with unknown grazing history, it is possible that only nonstressed algae with low resting levels of JA were surveyed. These resting levels might have been below the detection limit of our analytical method. Therefore, we performed a series of experiments where metabolic profiles of JA-,

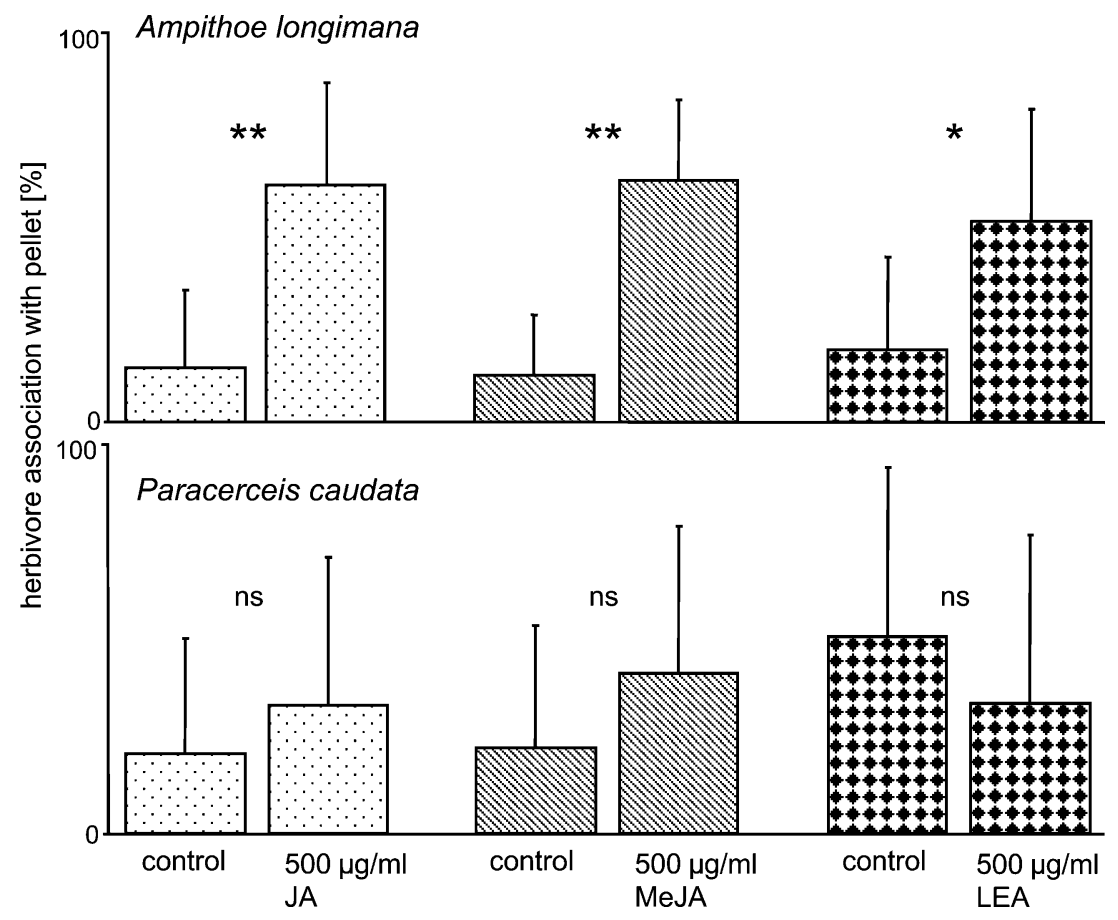

Fig. 3 Food selection by the amphipod Ampithoe longimana (top) and the isopod Paracerceis caudata (bottom). Mean percentage (+SD) of individuals present on food items. Choice assays were performed with artificial food derived from control alga (Dictyota dichotoma) and Dictyota dichotoma after jasmonic acid $(J A)$ incubation, methyl jasmonate $(M e J A)$ incubation, and linolenic acid ( $L E A)$ incubation. Number of replicates $N=21$ Ampithoe longimana/JA, $N=22$ Ampithoe longimana/MeJA, $N=24$ Ampithoe longimana/LEA, $N=24$ for all Paracerceis caudata experiments. $* * P=0.01, * P=0.05$, ns not significant 
MeJA-, and LEA-treated algae were compared to control samples. We selected a concentration range of these potential inducing reagents starting with amounts similar to those used for the induction of higher plants $\left(1,000 \mu \mathrm{g} \mathrm{ml}^{-1}\right.$; Boland et al. 1995). This comprehensive metabolic profiling enabled us to monitor changes of most volatiles, semivolatiles, acids, labile aldehydes, lipids, and other compound classes detectable by GC/MS and LC/MS. We did not aim to characterize all detected metabolites but rather we followed protocols established for the metabolic profiling of plant tissues that reveal global changes in metabolic profiles (Weckwerth et al. 2005; Shulaev et al. 2008). Accordingly, we did not bias towards specific compound classes but rather surveyed all accessible metabolites of the algae. Due to methodological limitations, we did not focus on phlorotannins, a dominant compound class in brown algae, often associated with chemical defense (e.g., Pavia and Toth 2000; Koivikko et al. 2008). In cases where a regulation of metabolite production was observed in response to the treatment, we verified the structures. In other cases, we relied on MS libraries to characterize compound classes but not exact structures.

This procedure gave no evidence for an induced up- or down-regulation of biosynthetic pathways with levels of inducing agents below $500 \mu \mathrm{g} \mathrm{ml}^{-1}$ of the potential hormones. This lack of secondary metabolite induction was found consistently in all investigated brown algal species. In similar induction experiments with higher plants as a positive control, identical analytical methods can be routinely used to monitor metabolic changes that manifest as dramatic change of metabolic profiles (see, e.g., Boland et al. 1995; Schüler et al. 2004). Since we did not observe any increase of amounts of JA in the tissue of brown algae, this value of $500 \mu \mathrm{g} \mathrm{ml}^{-1}$ medium is high and should be sufficient for induction if hormone-mediated processes were involved.

The employed hormone concentrations definitely exceed those required to trigger plant responses (Heil 2004), but we selected them to overcome dilution effects, since in the case of algae it is impossible to apply the inducing agents via vascular transport as is often the practice with higher plants. To avoid potential interferences of artificially elevated concentrations of putative hormones, a series of experiments also was run with $10 \mu \mathrm{g} \mathrm{ml}^{-1}$ of JA, MeJA, and LEA, respectively. These treatments did also not result in any change of the metabolic profile (data not shown).

The role of JA in the induction of terpene biosynthesis is well established for higher plants (Hopke et al. 1994; Keinanen et al. 2001), but similar processes were not observed in $D$. dichotoma as no change in the terpene profile was observed after treatments with the potential elicitors. The group of $D$. dichotoma metabolites that belong to diterpenes was identified by comparison with characteristic EI mass spectra from the literature, but no exact structures were assigned (Fattorusso et al. 1976; Teixeira et al. 2001; Barbosa et al. 2004; Kim et al. 2006). Additional experiments were performed with this alga by using coronalone as a JA analogue (Schüler et al. 2004). Coronalone mimics the action of coronatine, which is known to induce JA-like up-regulation of the production of terpenes in higher plants (Boland et al. 1995). We used coronalone in an additional bioassay to elucidate whether JA-responsive induction of secondary metabolites can be observed with a non-natural elicitor. It might be possible that coronalone is taken up more readily by the algae than JA. In addition, coronalone may not be subjected to the same (bacterial) transformation as JA if administered in the culture medium (Schüler et al. 2004). This JA analogue, which proved to be even more active than the hormone in experiments with higher plants, also had no effect on the absolute and relative concentration of $D$. dichotoma terpenoids.

Unspecific stress on the algae due to exposure to higher concentrations of the elicitors $\left(500-1,000 \mu \mathrm{g}\right.$ elicitor $\mathrm{ml}^{-1}$ seawater) over prolonged time spans led to the release of free fatty acids (Fig. 2). This was found for all investigated species with the exception of $S$. muticum. The metabolic responses were unspecific and were observed with the JA and MeJA treatments as well as with the LEA control. It is likely that increased stress causes activation of lipases, thus releasing fatty acids from (storage) lipids. Whereas saturated fatty acids, such as lauric (C12:0), myristic (C14:0), stearic (C18:0), arachidic (C20:0), and behenic acid (C22:0), and unsaturated fatty acids, such as stearidonic (C18:4), linolenic (C18:3), arachidonic (C20:4), and eicosapentaenoic acid (C20:5), were up-regulated occasionally, and only palmitic (C16:0), palmitoleic (C16:1), and oleic acid (C18:1) were found in all induction experiments with elevated amounts of phytohormones (Fig. 2). Because release of fatty acids was observed after all treatments (JA, MeJA, and LEA), we exclude this result as a specific hormonal action.

Comparative bioassays with induced and non-induced (control) D. dichotoma were performed to verify whetherdespite the lack of a detectable metabolic reaction - a more subtle up-regulation of chemical defense can be triggered by JA, MeJA, or the control treatment with LEA. The amphipod A. longimana and the isopod $P$. caudata served as model herbivores that are widely distributed along the Atlantic coast and that can be monitored closely under controlled conditions in the lab. Bioassay procedures to monitor defense reactions of brown algae that are due to medium- and non-polar metabolites as well as to volatiles are suitable, but they have not been verified for phlorotannins (results in Wiesemeier et al. 2007). No significant food preference was detected in assays where $P$. caudata had the choice between artificial foods derived from an 
induced or uninduced algae. In contrast, A. longimana preferred food from JA-, MeJA-, and LEA-treated algae, thereby contradicting the concept of induced defense. Interestingly, all three treatments (JA, MeJA, and LEA) led to weakly significant but comparable responses (Fig. 3). The lack of a specific induction of JA or MeJA and the fact that algae were highly stressed due to the elevated concentrations of the inducing reagents suggests that an unspecific stress response that weakens the algae might be detected by the herbivores. Since feeding by A. longimana is known to induce chemical defense in the closely related algal species Dictyota menstrualis (Cronin and Hay 1996), it thus has been documented that this amphipod is susceptible to induced brown algal chemical defense. Our results show that treatment with test compounds did not trigger the same chemical defense induction as feeding by the herbivore itself. Hormones or regulative principles other than JA and MeJA must, therefore, be responsible for the observed reaction (Cronin and Hay 1996).

The preference of treated, heavily stressed food by $A$. longimana might be due to the amphipod's ability to detect chemical cues of stressed algae that do not invest in chemical defense. Interestingly, the up-regulation of free fatty acids did not result in reduced food palatability, as has been observed in the interactions of grazers on epilithic biofilms (Jüttner 2001), thus indicating a resistance of these herbivores against free fatty acids that are lipolytic or deterrent in other alga-herbivore interactions (Fu et al. 2004).

In summary, we could not detect any indication for a role of JA and related metabolites in induced chemical defense of brown algae. The study covered a broad range of possible metabolic responses, but did not focus on the regulation of polyphenolics, which do play documented roles in induced chemical defense of some brown algae (Pavia and Toth 2000). We suggest that for the classes of metabolites investigated in our study, fundamentally different mechanisms for induction may have developed in higher plants and brown algae. Whether these mechanisms rely on structurally different hormones or on other regulative principles can currently not be ascertained.

Acknowledgments We thank Akira Peters and Florian Weinberger for their help with algal collection and identification. We are grateful to Marc Hay, who provided us with test animals and introduced us to the techniques for bioassays. Paulina Dabrowska and Birgit Schulze are acknowledged for help with analysis of phytohormones. We acknowledge the helpful support from Wilhelm Boland and funding from the Volkswagenstiftung.

\section{References}

Adra, C., Borgogni, A., and MarucchinI, C. 2006. Quantification of jasmonic acid by SPME in tomato plants stressed by ozone. J. Agric. Food Chem. 54:9317-9321.
Arnold, T. M., TARgett, N. M., TAnNer, C. E., Hatch, W. I., and FERRARI, K. E. 2001. Evidence for methyl jasmonate-induced phlorotannin production in Fucus vesiculosus (Phaeophyceae). J. Phycol. 37:1026-1029.

BALDWIN, I. T., Kessler, A., and HalitschKe, R. 2002. Volatile signaling in plant-plant-herbivore interactions: what is real? Curr. Opin. Plant Biol. 5:351-354.

Barbosa, J. P., Teixeira, V. L., and Pereira, R. C. 2004. A dolabellane diterpene from the brown alga Dictyota pfaffii as chemical defense against herbivores. Bot. Mar. 47:147-151.

Boland, W., Hopke, J., Donath, J., Nuske, J., and Bublitz, F. 1995. Jasmonic acid and coronatin induce odor production in plants. Angew. Chem. Int. Ed. 34:1600-1602.

Bouarab, K., Adas, F., Gaquerel, E., Kloareg, B., Salaun, J. P., and Potin, P. 2004. The innate immunity of a marine red alga involves oxylipins from both the eicosanoid and octadecanoid pathways. Plant Physiol. 135:1838-1848.

Christov, C., Pouneva, I., BozhKova, M., Toncheva, T., Fournadzieva, S., and ZAFIROVA, T. 2001. Influence of temperature and methyl jasmonate on Scenedesmus incrassulatus. Biol. Plant. 44:367371.

Cronin, G., and HaY, M. E. 1996. Induction of seaweed chemical defenses by amphipod grazing. Ecology 77:2287-2301.

Cruz-Rivera, E., and HaY, M. E. 2003. Prey nutritional quality interacts with chemical defenses to affect consumer feeding and fitness. Ecol. Monogr. 73:483-506.

CZerpak, R., Piotrowska, A., and SZULeCKA, K. 2006. Jasmonic acid affects changes in the growth and some components content in alga Chlorella vulgaris. Acta Physiol. Plant 28:195-203.

Fattorusso, E., Magno, S., Mayol, L., Santacroce, C., Sica, D., Amico, V., Oriente, G., Piattelli, M., and Tringali, C. 1976. Dictyol A and B, two novel diterpene alcohols from brown alga Dictyota dichotoma. J. Chem. Soc. Chem. Comm. 2:575-576.

FEUSSNER, I., and WASTERNACK, C. 2002. The lipoxygenase pathway. Annu. Rev. Plant Biol. 53:275-297.

Fu, M., Koulman, A., Van Rijssel, M., Lützen, A., Boer, M. K., TYL, M. R., and LIEBEZEIT, G. 2004. Chemical characterisation of three haemolytic compounds from the microalgal species Fibrocapsa japonica (Raphidophyceae). Toxicon 43:355-363.

Garcia-Brugger, A., Lamotte, O., Vandelle, E., Bourque, S., Lecourieux, D., Poinssot, B., Wendehenne, D., and Pugin, A. 2006. Early signaling events induced by elicitors of plant defenses. Mol. Plant-Microb. Interact. 19:711-724.

HeIL, M. 2004. Induction of two indirect defences benefits Lima bean (Phaseolus lunatus, Fabaceae) in nature. J. Ecol. 92:527-536.

Hemmi, A., Honkanen, T., and Jormalainen, V. 2004. Inducible resistance to herbivory in Fucus vesiculosus - duration, spreading and variation with nutrient availability. Mar. Ecol. Prog. Ser. 273:109-120.

Hopke, J., Donath, J., Blechert, S., and Boland, W. 1994. Herbivore-induced volatiles - the emission of acyclic homoterpenes from leaves of Phaseolus lunatus and Zea mays can be triggered by a beta-glucosidase and jasmonic acid. FEBS Lett. 352:146-150.

JÜTTNER, F. 2001. Liberation of 5,8,11,14,17-eicosapentaenoic acid and other polyunsaturated fatty acids from lipids as a grazer defense reaction in epilithic diatom biofilms. J. Phycol. 37:744-755.

KARBAN, R., and BALDWIN, I. T. 1997. Induced Responses to Herbivory. University of Chicago Press, Chicago, IL.

Keinanen, M., Oldham, N. J., and Baldwin, I. T. 2001. Rapid HPLC screening of jasmonate-induced increases in tobacco alkaloids, phenolics, and diterpene glycosides in Nicotiana attenuata. J. Agric. Food Chem. 49:3553-3558.

Kim, J. Y., Alamsjah, M. A., Hamada, A., Fujita, Y., and ISHIBASHI, F. 2006. Algicidal diterpenes from the brown alga Dictyota dichotoma. Biosci. Biotech. Biochem. 70:2571-2574. 
KoIVIKKo, R., ERANEN, J. K., and LOPONEN, J. 2008. Variation of phlorotannins among three populations of Fucus vesiculosus as revealed by HPLC and colorimetric quantification. J. Chem. Ecol. 34:57-64.

KRUPINA, M. V., and DATHE, W. 1991. Occurrence of jasmonic acid in the red alga Gelidium latifolium. Z. Naturforsch. C 46:1127-1129.

KupPer, F. C., Muller, D. G., and Peters, A. F. 2002. Oligoalginate recognition and oxidative burst play a key role in natural and induced resistance of sporophytes of laminariales. J. Chem. Ecol. 28:2057-2081.

Macaya, E. C., Rothausler, E., Thiel, M., Molis, M., and Wahl, M. 2005. Induction of defenses and within-alga variation of palatability in two brown algae from the northern-central coast of Chile: effects of mesograzers and UV radiation. J. Exp. Mar. Biol. Ecol. 325:214-227.

MithöFER, A., WANNER, G., and BOLAND, W. 2005. Effects of feeding Spodoptera littoralis on lima bean leaves. II. Continuous mechanical wounding resembling insect feeding is sufficient to elicit herbivory-related volatile emission. Plant Physiol. 137:1160-1168.

Molis, M., Korner, J., Ko, Y. W., KiM, J. H., and WAHL, M. 2006. Inducible responses in the brown seaweed Ecklonia cava: the role of grazer identity and season. J. Ecol. 94:243-249.

PaviA, H., and Tотн, G. B. 2000. Inducible chemical resistance to herbivory in the brown seaweed Ascophyllum nodosum. Ecology $81: 3212-3225$

PAVIA, H., Toth, G. B., and ABERG, P. 2002. Optimal defense theory: elasticity analysis as a tool to predict intraplant variation in defenses. Ecology 83:891-897.

POHNERT, G. 2004. Chemical defense strategies of marine organisms. Top. Curr. Chem. 239:179-219.

Schulze, B., Lauchli, R., Sonwa, M. M., Schmidt, A., and BOLAND, W. 2006. Profiling of structurally labile oxylipins in plants by in situ derivatization with pentafluorobenzyl hydroxylamine. Anal. Biochem. 348:269-283.

Schüler, G., MithöFer, A., BAldwin, I. T., Berger, S., Ebel, J., Santos, J. G., Herrmann, G., Hölscher, D., Kramell, R., Kutchan, T. M., Maucher, H., Schneider, B., Stenzel, I., WASTERNACK, C., and BOLAND, W. 2004. Coronalon: a powerful tool in plant stress physiology. FEBS Lett. 563:17-22.

Shulaev, V., Cortes, D., Miller, G., and Mittler, R. 2008. Metabolomics for plant stress response. Physiol. Plant. 132:199208.

Tarakhovskaya, E. R., Maslov, Y. I., and Shishova, M. F. 2007. Phytohormones in algae. Russ. J. Plant Physiol. 54:163-170.

TeiXeira, V. L., Cavalcanti, D. N., and Pereira, R. C. 2001. Chemotaxonomic study of the diterpenes from the brown alga Dictyota menstrualis. Biochem. Sys. Ecol. 29:313-316.

WASTERNACK, C. 2007. Jasmonates: An update on biosynthesis, signal transduction and action in plant stress response, growth and development. Ann. Bot. 100:681-697.

Weckwerth, W., and Morgenthal, K. 2005. Metabolomics: from pattern recognition to biological interpretation. Drug Discov. Today 10:1551-1558.

Wichard, T., Poulet, S. A., and Pohnert, G. 2005. Determination and quantification of alpha, beta, gamma, delta-unsaturated aldehydes as pentafluorobenzyl-oxime derivates in diatom cultures and natural phytoplankton populations: application in marine field studies. J. Chrom. B. 814:155-161.

Wiesemeier, T., Hay, M. E., and PoHNERT, G. 2007. The potential role of wound-activated volatile release in the chemical defence of the brown alga Dictyota dichotoma: blend recognition by marine herbivores. Aquat. Sci. 69:403-412.

WitTSTOCK, U., and GERSHENZON, J. 2002. Constitutive plant toxins and their role in defense against herbivores and pathogens. Curr. Opin. Plant Biol. 5:300-307. 